
2 Research Square
Preprints are preliminary reports that have not undergone peer review.
They should not be considered conclusive, used to inform clinical practice,
or referenced by the media as validated information.

\title{
The Expression and Prognostic Significance of the Nicotinic Receptor Cluster on 15q25 about CHRNA5 and PSMA4 mRNA in Lung Adenocarcinoma Utilizing TCGA Datasets
}

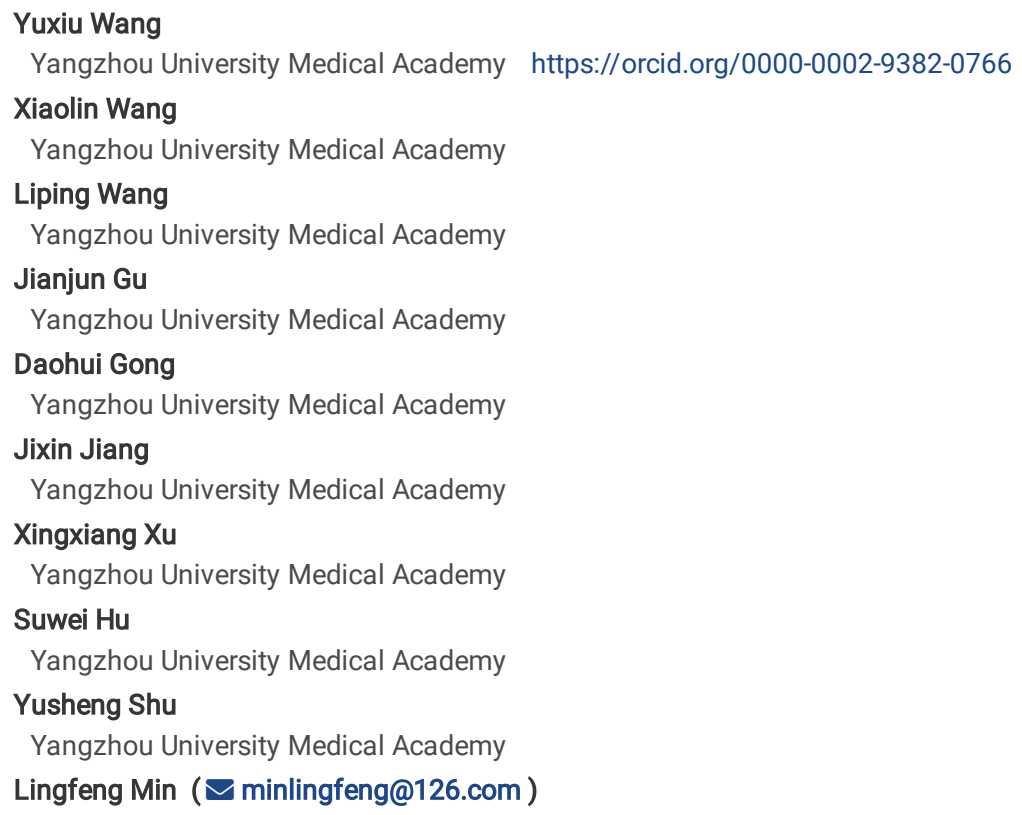




\section{Abstract}

Background: To explore the mutations expression and prognostic significance of 15q25 (CHRNA5 and PSMA4) mRNA in lung adenocarcinoma (LAC) based on immunohistochemistry, TCGA and bioinformatics. Methods: Mutations expression on chromosome 15q25 of 576 primary LAC patients was selected. And their survival and gene expression data were extracted from TCGA. The cell experiment about Beas-2b, A549 and H1299 cell lines were done to further prove the CHRNA5 and PSMA4 expression difference between lung cancer and normal cells. Immunohistochemistry data of CHRNA5 and PSMA4 were detected in LAC and normal tissues from 122 patients.Finally, Gene enrichment analysis (GSEA) was used to predict the regulatory genes of CHRNA5 and PSMA4.Results: CHRNA5 and PSMA4 are frequently mutated in the TCGA (CHRNA5, 1.7\%; PSMA4, 1.3\%). The expression of CHRNA5 and PSMA4 were obviously higher in A549 and H1299 cells. Immunohistochemical staining revealed that the level of CHRNA5 and PSMA4 was considerably higher in LAC group than in normal group. There was a significant association between high expression of CHRNA5 and Smoking History $(P=0.011)$, Smoking History Pack Year Value( $P=0.010)$. Besides, there was a significant correlation between CHRNA5 and PSMA4 expression level and prognosis $(P=0.003 ; P=0.008)$, and the higher expression, the worse prognosis. GSEA results suggested that CHRNA5 and PSMA4 high expression samples were respectively enriched to cell cycle, base excision repair, oxidative phosphorylation, protein export, aminoacyl tRNA biosynthesis etc.Conclusions: The expression of CHRNA5 and PSMA4 mRNA has a significant impact on survival of LAC, they may be a potential target for treating patients with lung adenocarcinoma.

\section{Background}

Lung cancer is the most common malignant tumor with highest morbidity and mortality worldwide, $80 \%$ of which are Non-Small Cell Lung Cancer (NSCLC). Lung adenocarcinomas ( $\mathrm{LAC}$ ) is the most common pathology subtype in lung cancer [1]. More and more evidence revealed that multigene mutations also do contribute to the risk of carcinogenesis and may have a detrimental role in an individual's susceptibility to LAC[2]. Although the therapy of LAC has made little progress at the early stage, most patients are at later stage, and there is no effective treatment for these patients, the 5 -year overall survival rate is only $10 \%$ to $15 \%$ [3]. Genetics, now as a novel therapy for advanced LAC patients, has acquired favorable curative effect and significant prolonged the LAC patient's survival time [4]. It is urgent to find effective prognostic biomarkers for LAC.

Epidemiological data show that there is a close connection between in the occurrence, development of lung cancer and lots of environmental factors, such as smoking behaviors [5], air pollution [6], occupation [7] and so on. Nicotine is one of the main psychoactive ingredients in tobacco that contributes to the harmful tobacco smoking habit [8]. A common feature of addictive drugs, including nicotine, is that they increase dopamine (DA) release in the nucleus accumbens (NAc). The principal dopaminergic projections to NAc arise from neurons in the ventral tegmental area (VTA). In the VTA, nicotine acts as an agonist to activate and desensitize these nicotinic acetylcholine receptors (nAChRs), that facilitated the reinforcing effects of nicotine [9]. More recently, some large genome-wide association studies(GWAS) identified an association between single nucleotide polymorphism (SNP) variation at 15q24-15q25.1 and susceptibility in lung cancer [10]. IREB2, CHRNA5, CHRNA3, CHRNB4, HYKK and PSMA4 were located on chromosome 1525.1 gene cluster which signaling pathways involved in nicotine metabolism and addiction. Smoking for a while there's relativity between peripheral blood nicotine levels, and gene polymorphism gene cluster in nicotine metabolism existed obvious correlation, and case fatality rate of obvious correlation with smoking related diseases, including lung cancer[11], chronic obstructive pulmonary disease (COPD) [12], etc.

Recently genome-wide association studies of lung cancer have shown common variation at 15q24-25.1 as a determinant of risk. Results of genetic association studies for nicotine dependence, smoking behavior, and smoking related diseases have converged to implicate the chromosome $15 \mathrm{q} 25.1$ region. Therefore, this study explored the effect of IREB2, CHRNA5, CHRNA3, CHRNB4, HYKK and PSMA4 gene cluster expression in LAC on prognosis survival utilizing TCGA datasets, looking for a highly sensitive molecular marker. It can provide clues and ideas for the early diagnosis and prognosis of LAC.

\section{Methods}

\section{TCGA data collection}

Using Bioconductor packages/TCGA biolinks into the TCGA Research Network (http://cancer genome.nih.gov/) to download and preprocessing data, a total of seven cohort with lung adenocarcinoma related research. They are the Broad, MSKCC, Nature, PanCancer, Provisional, TSP and MSK-IMPACT cohort respectively, with a total of 3232 samples. According to the integrity of gene sequence, copy number variation and smoking history, finally Provisional cohort eventually conformed to our research criteria. The mutation of IREB2, CHRNA5, CHRNA3, CHRNB4, HYKK and PSMA4 gene in LAC were generated from

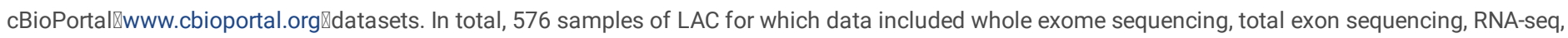
microRNA-seq, protein expression, gene expression and CNA data. At the same time, we also downloaded the clinical data, such as patient Age, Gender, Race, Smoking History, Tumor staging, Metastasis, and Survival for clinicopathological correlation analysis and prognosis analysis.

\section{Cell culture}

The cell lines Beas-2b, A549 and H1299 were purchased from Shanghai institute of life sciences. The culture conditions were 1640 containing $10 \%$ fetal bovine serum training based on wet $37^{\circ} \mathrm{C}$ and $5 \% \mathrm{CO} 2$, constant temperature incubator culture cells.

\section{Patients and tissues collection}

Sixty FFPE samples with normal and carcinoma tissues samples were obtained from patients with LAC at Biobank of Northern Jiangsu People's Hospital (Yangzhou, China) between July 2015 and December 2017. All tumor tissues were histologically reviewed by two pathologists. Patients signed informed consent, and study protocols for the use of human tissues were approved by and performed in accordance with the ethical standards of the Ethics Committee of the Northern Jiangsu People's Hospital. 
Four-micron-thick sequential histological tumor sections were obtained from a representative formalin-fixed, paraffin-embedded tumor block and used for immunohistochemistry (IHC) analysis. Polyclonal rabbit anti-CHRNA5 antibody (Abcam, UK) was used as the primary antibody at a dilution level of 1:50 incubated for stay overnight at $4^{\circ} \mathrm{C}$, HYKK used Polyclonal rabbit anti-HYKK antibody (Abgent, USA) at a dilution level of 1:50 and PSMA4 used Polyclonal rabbit anti-PSMA4 antibody (Origenes, USA) at a dilution level of 1:25. Polyclonal goat anti-HRP antibody (ZSGB, China) was used as the second antibody. To measure the IHC expression of the different markers and quantify the inflammatory cells expressing, the slides, containing whole tumor sections, were digitally scanned at ×200 magnification. The images were visualized and analyzed as described in Supplementary Methods.

\section{Western blotting}

Total protein concentration was measured by bicinchoninic acid assay. Protein $(60 \mu \mathrm{g})$ was separated by $10 \%$ SDSPAGE and subsequently transferred onto polyvinylidene difluoride membranes. Following blocking with $5 \%$ bovine serum albumin, the membrane was incubated with the appropriate primary antibody at $4^{\circ} \mathrm{C}$ overnight, followed by washing and incubation with horseradish peroxidase goatantirabbit Immunoglobulin $\mathrm{G}$ secondary antibody for $1 \mathrm{~h}$ at room temperature and detection using an enhanced chemiluminescence kit. Western blots were performed using Polyclonal rabbit anti-CHRNA5 antibody (Abcam, 1:500), HYKK used Polyclonal rabbit anti-HYKK antibody (Abgent, 1:250) and PSMA4 used Polyclonal rabbit anti-PSMA4 antibody (Origenes, 1:50) and the GAPDH specific polyclonal antibody (KC-5G4; Kangcheng Shanghai, China). The images were captured using the Gel Dox XR system (Bio-Rad, Philadelphia, PA).

\section{Gene set enrichment analysis}

GSEA 2.2.1 software was used for enrichment analysis. According to the expression level of CHRNA5 and PSMA4 which divided into high expression group and low expression group. In this study, we obtained c2.cp.cp. kegg.v5.1 Symbols.GMT datasets from the MsigDB database of GSEA website. Then, we by default weighted enrichment statistics method for enrichment analysis, the number of random combinations was set to 1,000 times.

\section{Statistical analyses}

All statistical analyses were performed using the SPSS 22.0 software package. The association between CHRNA5 and PSMA4 expression and clinicopathological features was analyzed using the $\chi 2$ test. Kaplan-Meier curves and the log-rank test were used to analyze the survival data. $P<0.05$ was considered statistically significant. All values are reported as the mean $\pm \mathrm{SE}$.

\section{Results}

The mutation of IREB2, CHRNA5, CHRNA3, CHRNB4, HYKK and PSMA4 in LAC based on the TCGA database.

In total, 576 patients with a histological confirmed diagnosis of LAC were collected by TCGA in the present study, the main data of which contain the whole exome sequencing, RNA-seq, copy number, gene expression and CNA data. The individual mutation frequencies of Privisional cohort are illustrated in Figure 1. IREB2 was mutated in 7\% of tumors, 41 of the 576 sequenced patients had IREB2 mutations; CHRNA5 was mutated in $5 \%$ of tumors, 30 of the 576 sequenced patients had CHRNA5 mutations; CHRNA3 was mutated in 5\% of tumors, 30 of the 576 sequenced patients had CHRNA3 mutations; CHRNB4 was mutated in $7 \%$ of tumors, 41 of the 576 sequenced patients had CHRNB4 mutations; As well as HYKK in $6 \%$ and PSMA4 with a mutation rate of $9 \%$, altered in 35 and 53 of 576 sequenced patients.

Association between the expression of IREB2, CHRNA5, CHRNA3, CHRNB4, HYKK and PSMA4 and clinical pathological features based on the TCGA database.

To understand the role of IREB2, CHRNA5, CHRNA3, CHRNB4, HYKK and PSMA4 in the tumorigenesis and progression in LAC, the study analyzed the association between the expression of IREB2, CHRNA5, CHRNA3, CHRNB4, HYKK and PSMA4 and clinicopathological features. The LAC patients were divided into two groups by the expression of IREB2, CHRNA5, CHRNA3, CHRNB 4, HYKK and PSMA4, based on the median value, namely, low expression group and high expression group. In this reseach, there was a significant association between a high expression of CHRNA5 and PSMA4 and Gender $(P=0.000, P=$ $0.005)$, , overall survival status $(P=0.050, P=0.006)$ and recurrence free survival status $(P=0.019, P=0.049)$. High expression of CHRNA5 and Smoking $(P=$ 0.002), , as shown in Table 1. However, the expression of CHRNA5 and PSMA4 was not associated with age $(P=0.855)$," race $(P=0.752)$," TNM Stage $(P=$ $0.725)$ or Disease Stage $(P=0.971)$ in the cohort $(P>0.05)$..

\section{Evaluation of IREB2, CHRNA5, CHRNA3, CHRNB4, HYKK and PSMA4 gene expression in LAC tissues based on the TCGA database.}

We examined the lung expression of IREB2, CHRNA5, CHRNA3, CHRNB4, HYKK and PSMA4 in normal and carcinoma tissues two experiment groups based on the TCGA database. The result showed that CHRNA5, CHRNB4, HYKK, and PSMA4 were highly expressed in lung adenocarcinoma tissues, and IREB2 and CHRNA3 were lowly expressed in the cancer tissues (Figure 2). In addition, the expression of IREB2, CHRNA5, CHRNA3, CHRNB4, HYKK and PSMA4 were significant different between normal and lung adenocarcinoma tissues $(P<0.001, P<0.001, P=0.021, P<0.001, P<0.001, P<0.001)($ Table 2$)$.

Association between the expression of CHRNA5, PSMA4 and survival based on the TCGA database.

The research used TCGA datasets and Kaplan-Meier Plotter online data respectively for survival analysis. Results showed that the expression level of CHRNA5 and PSMA4 was significantly correlated with the prognosis. There was a significant correlation between CHRNA5 and PSMA4 expression level and prognosis. There were significant differences in OS/RFS between the high and low CHRNA5, PSMA4 expression groups $(P<0.05)$.. The higher the expression, the worse the prognosis. Further utilization Kaplan-Meier Plotter online data analysis was shown in Fig. 3. But there were no difference in OS/RFS between the high and 
low IREB2 CHRNA3, CHRNB4, HYKK expression groups (shown in supplement Figure 2). The above results suggest that CHRNA5 and PSMA4 is an adverse prognostic factor and high expression was significantly associated with shorter survival.

The expression of CHRNA5 and PSMA4 in Beas-2b, A549 and H1299 cells.

To further demonstrate the role of CHRNA5 and PSMA4 expression in the development and progression of lung adenocarcinoma, we conducted a cell experiment to compare the expression of CHRNA5 and PSMA4 in normal and cancer cells. The result showed that the expression of CHRNA5 and PSMA4 in A549 and $\mathrm{H} 1299$ cells were obviously higher than beas-2b cell(Figure 4).

The expression of CHRNA5 and PSMA4 in tissue microarray of lung adenocarcinoma

Immunohistochemical staining revealed that the level of CHRNA5 and PSMA4 in the lung tissues of the carcinoma group was considerably higher than those in the normal group, the positive expression products were brown-yellow granules, which were located in nucleus and cytoplasm respectively (as shown in Figure 5).

The relationship between the expression levels of CHRNA5 and PSMA4 and the clinical characteristic of patients with lung adenocarcinoma.

In order to further explore the effect of CHRNA5 and PSMA4 in the clinical pathology of lung adenocarcinoma, the study analyzed the association between the expression of CHRNA5 and PSMA4 and clinicopathological features. The LAC patients were divided into two groups by the expression of CHRNA5 and PSMA4, based on the median value, namely, low expression group and high expression group. In this reseach, there was a significant association between a high expression of CHRNA5 and T stage $(P=0.026)$,, overall survival status $(P=0.007)$ and progressive free survival status $(P=0.013)$.. And there was a significant association between a high expression of PSMA4 and overall survival status $(P=0.006)$ and progressive free survival status $(P=0.007)$, as shown in Table 3. However, the expression of CHRNA5 and PSMA4 was not associated with age, race, TM Stage or Disease Stage in the cohort $(P>0.05)$..

Correlation between the expression levels of seven PSMAs and overall Survival (OS)/ recurrence-free survival (PFS)

And then we used the Kaplan-Meier for survival analysis of our clinical data. Results showed that the expression level of CHRNA5 and PSMA4 was significantly correlated with the prognosis. There was a significant correlation between CHRNA5 and PSMA4 expression level and prognosis. There were significant differences in OS/RFS between the high and low CHRNA5, PSMA4 expression groups $(P<0.05)$.. The higher the expression, the worse the prognosis (Figure 6).

The functional enriched analysis of CHRNA5, HYKK and PSMA4

The results of GSEA method suggested that CHRNA5, HYKK and PSMA4 high expression samples were respectively enriched to cell cycle, base excision repair, oxidative phosphorylation, protein export, aminoacyl tRNA biosynthesis, etc (see Figure 7).

\section{Discussion}

Over the past decades, the prognosis of LAC has been poor, and it still lacks a highly sensitive and specified diagnosis of early diagnosis of LAC. Among all therapies for $L A C$, surgery is the main treatment. If $L A C$ can be diagnosed at the early stage, the prognosis of LAC will be greatly improved. The previous studies found many tumor markers, but lots of them turned out to have little practical clinical value. Under these circumstances, it is urgent to find effective prognostic biomarkers for LAC. Recent studies showed that the gene polymorphism, the mRNA expression located on chromosome 15q25.1 was significantly correlated with human smoking behaviors, smoking amount and even smoking times. It was also significantly correlated with various studies. For example, strong risk loci on chromosome 15q25 for lung cancer in African-American population, the genome-wide significance $15 q 25.1$ marked by rs2036527 near CHRNA5[13]. Ji et al reported that genetic variants on the $15 q 25.1$ lung cancer susceptibility locus may influence susceptibility to NPC, particularly for smoking-associated nasopharyngeal carcinoma [14]. Single-nucleotide polymorphisms (SNPs) in the cholinergic nicotinic receptor subunit genes on chromosome 15q25.1, including CHRNA3, CHRNB4 and CHRNA5, are well-established biomarkers of chronic obstructive pulmonary disease (COPD) and lung cancer [15]. Several of the polymorphisms and their haplotypes in CHRNA5/CHRNA3 genes may have functional effects on (i) CHRNA5 mRNA levels, (ii) polycyclic aromatic hydrocarbon-DNA adduct levels, (iii) TP53 mutations and (iv) susceptibility to lung cancer [16]. However, the function of the nicotinic receptor cluster on chromosome $15 q 25$ in LAC is still unclear.

In this study, we used the TCGA databases to analyze the association of 15q25 locus genes with LAC. The results showed that the mRNA expression levels of CHRNA5 and PSMA4 were significantly up regulated in LAC compared with normal tissues. In addition, we also analyzed the relationship between the mRNA expression levels of CHRNA5 and PSMA4 and various clinicopathological features and survival outcomes of LAC based on the TCGA database. The results showed that there was a significant association between a high expression of CHRNA5 and PSMA4 and 0verall survival status and recurrence free survival status. However, the expression of CHRNA5 and PSMA4 were not associated with age, race, TNM Stage or Disease Stage in the cohort (P>0.05). Besides, survival curve analysis was also done, and the result showed that CHRNA5 and PSMA4 expression were significantly higher in patients with a reduced overall survival $(P<0.05)$ reduced recurrence free survival $(P<0.05)$. We believe that there is an obvious correlation between CHRNA5 and PSMA4 expression and the pathologic features and prognosis of LAC. All of the TCGA analysis results support the hypothesis that the change of CHRNA5 and PSMA4 expression may participate in the development of lung cancer, especially LAC.

In order to further demonstrate our hypothesis, we also used the cells experiments and clinical data to verify it. The results shown that the expression of CHRNA5 and PSMA4 in A549 and H1299 cells were obviously higher than beas-2b cell, and they were also widely expressed in the cells of LAC tissue samples, especially in the cytoplasm. In addition, our clinical data analysis has shown that there was a significant association between a high expression of

Page $4 / 14$ 
CHRNA5 and T stage $(P=0.026)$,, overall survival status $(P=0.007)$ and progressive free survival status $(P=0.013)$.. And there was a significant association between a high expression of PSMA4 and overall survival status $(P=0.006)$ and progressive free survival status $(P=0.007)$.. Moreover, There was a significant correlation between CHRNA5 and PSMA4 expression level and prognosis. There were significant differences in OS/RFS between the high and low CHRNA5, PSMA4 expression groups $(P<0.05)$.. The results above further support our hypothesis.

CHRNA proteins are expressed in lung epithelial cells and bind nicotine, an addictive compound in cigarettes, and nitrosamines, potential lung carcinogens in cigarettes and food $[17,18]$. Signal transduction through CHRNA proteins was suggested to cause cell proliferation and also to facilitate neoplastic transformation. Recently, the locus containing two genes encoding nicotine acetylcholine receptor (nAChR) subunits, CHRNA3 and CHRNA5, was shown to be associated with lung cancer risk in Caucasians by three genome-wide association (GWA) studies[17, 19, 20]. A decreased survival correlation with lung cancer has been shown because the reduced treatment efficacy and the worse survival may be well explained by the nAChRs pathway through CHRNA proteins on tumor cell proliferation, apoptosis, epithelial-mesenchymal transition, and proinvasive and angiogenic effects[21, 22]. Our results showed that CHRNA5 were higher expressed in lung adenocarcinoma tissues, and that there exists a significant association between the expression and clinical characteristics and prognosis of patients. As for upper result, CHRNA5 could be used as a potential prognostic marker and a therapeutic target in lung adenocarcinoma.

PSMA4 was also located at the 15q24-25.1 locus and encodes a structural protein of the 20S proteasome core and was required for proteasomal activities. The proteasome is responsible for the regulation of many cellular processes including transcription, cell cycle progression and apoptosis [23]. Proteasome dysfunction can lead to many diseases including cancer, and drugs inhibit proteasome activity directly affect lung cancer susceptibility through its modulation of cell proliferation and apoptosis [24]. Aberration and abnormal expression of proteasome subunits have been demonstrated in many tumors including breast cancer [25-27], lung cancer [28], hepatocellular carcinoma [29] and colorectal cancer [30]. PSMA4 is a component of the Adenosine Triphosphate- and ubiquitin-dependent nonlysosomal pathway, and it is involved in the processing of class I major histocompatibility complex peptides. It has been reported that proteasome subunit alpha type-4 (PSMA4)[31] mRNA levels were increased in lung tumors. In our results, we also found that PSMA4 were highly expressed in lung adenocarcinoma tissues, and showed a significant association between the expression and clinical characteristics and prognosis of patients. That further confirmed that PSMA4 plays an important role in the occurrence and development of lung adenocarcinoma.

However, the occurrence of lung cancer is a multi-step process involving multiple genes. How does the CHRNA5 and PSMA4 expression regulate the development of lung adenocarcinoma. What processes underlie this regulation, and how do they relate to one other? We used the GSEA method to predict the signal pathways in which they may participate. The result suggested that CHRNA5 and PSMA4 high expression samples were respectively enriched to cell cycle, base excision repair, oxidative phosphorylation, protein export, aminoacyl tRNA biosynthesis. And the specific mechanism of that remain require further study. Furthermore, methods of detecting the expression of the CHRNA5, HYKK and PSMA4 in the sera of patients, which would be of great value for clinical diagnosis, must be developed.

\section{Conclusions}

In summary, our study systematically analyzed the mRNA expression levels and prognostic significance of CHRNA5 and PSMA4 in lung adenocarcinoma. The CHRNA5 and PSMA4 exhibited significant expression differences between tumor and normal tissues. In addition, we further confirmed that CHRNA5 and PSMA4 were widely expressed in the cancer cells such as A549 and H1299 cells. Moreover, the expression of CHRNA5 and PSMA4 could significant affect the prognosis of LAD patients. However, the occurrence of lung cancer is a multi-step process involving multiple genes. How does the CHRNA5 and PSMA4 expression regulate the development of lung adenocarcinoma? What processes underlie this regulation, and how do they relate to one other? In future studies, we'll increase the sample volume to explore the detailed roles of the CHRNA5 and PSMA4 in tumor initiation and development, which may strengthen the evidence that the CHRNA5 and PSMA4 could be promising therapeutic targets and novel prognostic biomarkers for lung adenocarcinoma.

\section{Abbreviations}

LAC: lung adenocarcinoma; TCGA: The Cancer Genome Atlas; PSMA4: proteasome subunit alpha type-4; CHRNA5:Recombinant Cholinergic Receptor; HYKK: hydroxylysine kinase

\section{Declarations}

\section{Ethics approval and consent to participate}

All participants were voluntary and provided written informed consent before taking part in this research. This study was approved by the Ethics Committee of the Northern Jiangsu People's Hospital, and in compliance with the Declaration of Helsinki. The design and performance of this study involving human subjects were obviously described in a research protocol.

\section{Consent for publication}

No conflict of interest exits in the submission of this manuscript, and manuscript is approved by all authors for publication.

\section{Availability of data and material}

All the data regarding the findings are available within the manuscript. Anyone who is interested in the information should contact the corresponding author. 


\section{Competing interests}

The authors declare that they have no competing interests.

\section{Funding}

This work was supported by the National Natural Science Foundation of China [Grant numbers 81870033], the Six Talent Peaks Poject of Jiangsu Province [Grant number WSN-106], the Medical Scientific Research Foundation of Jiangsu Province of China [Grant numbers QNRC2016340], the Foundation for Highlevel Talents during the 13th Five-year Plan Period of Yangzhou, China [Grant numbers ZDRC201866].

Professor lingfeng Min, the recipient of these funds, was the principal administrator of this study.

\section{Authors' contributions}

Yuxiu Wang performed the histological examination of the lung tissues, and was a major contributor in writing the manuscript. Xiaolin Wang, Liping Wang and Jixin Jiang analyzed and interpreted the patient data. Jianjun Gu, Daohui Gong and Suwei Hu mainly performed the cell examination. Lingfeng Min, Xingxiang Xu and Shusheng Yu mainly implement the modification after writing of this article. All authors read and approved the final manuscript.

\section{Acknowledgments}

We acknowledge reviewers and academic editor for their constructive suggestions on our manuscript. We also thank the Biobank of Northern Jiangsu People's Hospital for providing us with the research samples.

\section{References}

1.Ray MA, Faris NR, Smeltzer MP, Fehnel C, Houston-Harris C, Levy P, Wiggins L, Sachdev V, Robbins T, Spencer D et al: Effectiveness of Implemented Interventions on Pathologic Nodal Staging of Non-Small Cell Lung Cancer. ANN THORAC SURG 2018, 106(1):228-234.

2.Wu M, Pan X, Xu Y, Wu S, Wu X, Chen B: Methodological comparison of the allele refractory mutation system and direct sequencing for detecting EGFR mutations in NSCLC, and the association of EGFR mutations with patient characteristics. ONCOL LETT 2018, 16(1):1087-1094.

3.Bracht J, Mayo-de-Las-Casas C, Berenguer J, Karachaliou N, Rosell R: The Present and Future of Liquid Biopsies in Non-Small Cell Lung Cancer: Combining Four Biosources for Diagnosis, Prognosis, Prediction, and Disease Monitoring. CURR ONCOL REP 2018, 20(9):70.

4.Wang Z, Cheng Y, An T, Gao H, Wang K, Zhou Q, Hu Y, Song Y, Ding C, Peng F et al: Detection of EGFR mutations in plasma circulating tumour DNA as a selection criterion for first-line gefitinib treatment in patients with advanced lung adenocarcinoma (BENEFIT): a phase 2, single-arm, multicentre clinical trial. Lancet Respir Med 2018, 6(9):681-690.

5.Minnix JA, Karam-Hage M, Blalock JA, Cinciripini PM: The importance of incorporating smoking cessation into lung cancer screening. Transl Lung Cancer Res 2018, 7(3):272-280.

6.Cao Q, Rui G, Liang Y: Study on PM2.5 pollution and the mortality due to lung cancer in China based on geographic weighted regression model. BMC PUBLIC HEALTH 2018, 18(1):925.

7.Jung J, Feinstein SG, Palma LL, Macleod JS, Arrandale VH, McLeod CB, Peter A, Demers PA: Examining lung cancer risks across different industries and occupations in Ontario, Canada: the establishment of the Occupational Disease Surveillance System. OCCUP ENVIRON MED 2018, 75(8):545-552.

8.Zhao S, Chen F, Wang D, Wang H, Han W, Zhang Y: Effect of preoperative smoking cessation on postoperative pain outcomes in elderly patients with high nicotine dependence. Medicine (Baltimore) 2019, 98(3):e14209.

9.0zga JE, Anderson KG: Reduction in delay discounting due to nicotine and its attenuation by cholinergic antagonists in Lewis and Fischer 344 rats. Psychopharmacology (Berl) 2018, 235(1):155-168.

10.Xun WW, Brennan P, Tjonneland A, Vogel U, Overvad K, Kaaks R, Canzian F, Boeing H, Trichopoulou A, Oustoglou E et al: Single-nucleotide polymorphisms (5p15.33, 15q25.1, 6p22.1, 6q27 and 7p15.3) and lung cancer survival in the European Prospective Investigation into Cancer and Nutrition (EPIC).

MUTAGENESIS 2011, 26(5):657-666.

11.Wen L, Jiang K, Yuan W, Cui W, Li MD: Contribution of Variants in CHRNA5/A3/B4 Gene Cluster on Chromosome 15 to Tobacco Smoking: From Genetic Association to Mechanism. MOL NEUROBIOL 2016, 53(1):472-484.

12.Ellerbeck EF, Nollen N, Hutcheson TD, Phadnis M, Fitzgerald SA, Vacek J, Sharpe MR, Salzman GA, Richter KP: Effect of Long-term Nicotine Replacement Therapy vs Standard Smoking Cessation for Smokers With Chronic Lung Disease: A Randomized Clinical Trial. JAMA Netw Open 2018, 1(5):e181843. 
13. Huang CY, Xun XJ, Wang AJ, Gao Y, Ma JY, Chen YT, Jin TB, Hou P, Gu SZ: CHRNA5 polymorphisms and risk of lung cancer in Chinese Han smokers. AM J CANCER RES 2015, 5(10):3241-3248.

14.Anantharaman D, Chabrier A, Gaborieau V, Franceschi S, Herrero R, Rajkumar T, Samant T, Mahimkar MB, Brennan P, McKay JD: Genetic variants in nicotine addiction and alcohol metabolism genes, oral cancer risk and the propensity to smoke and drink alcohol: a replication study in India. PLOS ONE2014, 9(2):e88240.

15.Zhao Z, Zhou Y, Li Y, Jiang C, Zhao D, Zhao Z, Liu Z, Ran P: An efficient method to genotype the polymorphisms of cholinergic nicotinic receptor subunit genes and their associations with COPD onset risk. EXP LUNG RES 2016, 42(5):267-274.

16.Tekpli X, Landvik NE, Skaug V, Gulsvik A, Haugen A, Zienolddiny S: Functional effect of polymorphisms in $15 q 25$ locus on CHRNA5 mRNA, bulky DNA adducts and TP53 mutations. INT J CANCER 2013, 132(8):1811-1820.

17.Berrettini WH, Doyle GA: The CHRNA5-A3-B4 gene cluster in nicotine addiction. Mol Psychiatry 2012, 17(9):856-866.

18.Shen B, Zhu Q, Zheng MQ, Chen J, Shi MQ, Feng JF: CHRNA5 polymorphism and susceptibility to lung cancer in a Chinese population. BRAZ J MED BIOL RES 2013, 46(1):79-84.

19.Yoo SS, Kang HG, Choi JE, Do SK, Lee WK, Choi SH, Lee SY, Lee SY, Lee J, Cha SI et al: Effects of polymorphisms identified in genome-wide association studies of never-smoking females on the prognosis of non-small cell lung cancer. Cancer Genet 2017, 212-213:8-12.

20.Zanetti KA, Wang Z, Aldrich M, Amos Cl, Blot WJ, Bowman ED, Burdette L, Cai Q, Caporaso N, Chung CC et al: Genome-wide association study confirms lung cancer susceptibility loci on chromosomes 5p15 and 15q25 in an African-American population. LUNG CANCER 2016, 98:33-42.

21. Hallden S, Sjogren M, Hedblad B, Engstrom G, Hamrefors V, Manjer J, Melander O: Gene variance in the nicotinic receptor cluster (CHRNA5-CHRNA3CHRNB4) predicts death from cardiopulmonary disease and cancer in smokers. J INTERN MED 2016, 279(4):388-398.

22.Wang Y, Wu H, Liu Q, Wang C, Fu L, Wang H, Zhu W, Fu W, Lv Y, Wang S et al: Association of CHRNA5-A3-B4 variation with esophageal squamous cell carcinoma risk and smoking behaviors in a Chinese population. PLOS ONE 2013, 8(7):e67664.

23.Cron KR, Zhu K, Kushwaha DS, Hsieh G, Merzon D, Rameseder J, Chen CC, D'Andrea AD, Kozono D: Proteasome inhibitors block DNA repair and radiosensitize non-small cell lung cancer. PLOS ONE 2013, 8(9):e73710.

24.Ling YH, Liebes L, Zou Y, Perez-Soler R: Reactive oxygen species generation and mitochondrial dysfunction in the apoptotic response to Bortezomib, a novel proteasome inhibitor, in human H460 non-small cell lung cancer cells, vol. 278; 2003.

25.Song H, Xiong H, Che J, Xi QS, Huang L, Xiong HH, Zhang P: Gel-based chemical cross-linking analysis of 20S proteasome subunit-subunit interactions in breast cancer. J Huazhong Univ Sci Technolog Med Sci 2016, 36(4):564-570.

26.Lagadec C, Vlashi E, Frohnen P, Alhiyari Y, Chan M, Pajonk F: The RNA-binding protein Musashi-1 regulates proteasome subunit expression in breast cancer- and glioma-initiating cells. STEM CELLS 2014, 32(1):135-144.

27.Okumura T, Ikeda K, Ujihira T, Okamoto K, Horie-Inoue K, Takeda S, Inoue S: Proteasome 26S subunit PSMD1 regulates breast cancer cell growth through p53 protein degradation. J BIOCHEM 2018, 163(1):19-29.

28.Taromi S, Lewens F, Arsenic R, Sedding D, Sanger J, Kunze A, Mobs M, Benecke J, Freitag H, Christen F et al: Proteasome inhibitor bortezomib enhances the effect of standard chemotherapy in small cell lung cancer. Oncotarget 2017, 8(57):97061-97078.

29.Chen YJ, Wu H, Shen XZ: The ubiquitin-proteasome system and its potential application in hepatocellular carcinoma therapy. CANCER LETT 2016, 379(2):245-252.

30.Wu T, Chen W, Zhong Y, Hou X, Fang S, Liu CY, Wang G, Yu T, Huang YY, Ouyang X et al: Nuclear Export of Ubiquitinated Proteins Determines the Sensitivity of Colorectal Cancer to Proteasome Inhibitor. MOL CANCER THER 2017, 16(4):717-728.

31.Wang T, Chen T, Thakur A, Liang Y, Gao L, Zhang S, Tian Y, Jin T, Liu JJ, Chen M: Association of PSMA4 polymorphisms with lung cancer susceptibility and response to cisplatin-based chemotherapy in a Chinese Han population. Clinical and Translational Oncology 2015, 17(7):564-569.

\section{Tables}

Table 1: The association between the expression of IREB2, CHRNA5, CHRNA3, CHRNB4, HYKK and PSMA4 and clinical pathological features based on the TCGA database. 


\begin{tabular}{|c|c|c|c|c|c|c|c|c|c|c|c|c|c|c|c|c|}
\hline \multirow[t]{2}{*}{ Characteristics } & \multirow[t]{2}{*}{$\mathrm{n}$} & \multicolumn{2}{|l|}{ IREB 2} & \multirow[t]{2}{*}{$\mathrm{X}^{2}$} & \multirow[t]{2}{*}{$P$} & \multicolumn{2}{|l|}{ CHRNA5 } & \multirow[t]{2}{*}{$\mathrm{X}^{2}$} & \multirow[t]{2}{*}{$\mathrm{P}$} & \multicolumn{2}{|l|}{ CHRNA3 } & \multirow[t]{2}{*}{$\mathrm{X}^{2}$} & \multirow[t]{2}{*}{$P$} & \multicolumn{2}{|l|}{ CHRNB4 } & z \\
\hline & & Low[288] & High[288] & & & Low[281] & High[286] & & & Low[275] & High[282] & & & Low[279] & High[278] & \\
\hline \multicolumn{17}{|l|}{ Gender } \\
\hline Female & 310 & 163 & 147 & 1.788 & 0.` 105 & 176 & 112 & 12.322 & 0.000 & 156 & 132 & 0.028 & 0.467 & 157 & 131 & ( \\
\hline Male & 266 & 125 & 141 & & & 134 & 154 & & & 154 & 134 & & & 153 & 135 & \\
\hline \multicolumn{17}{|l|}{ Age } \\
\hline$<40$ & 177 & 98 & 79 & 3.622 & 0.164 & 95 & 82 & 1.797 & 0.407 & 93 & 84 & 1.054 & 0.590 & 92 & 85 & ( \\
\hline $40-60$ & 346 & 163 & 183 & & & 167 & 179 & & & 166 & 180 & & & 171 & 175 & \\
\hline$>60$ & 34 & 15 & 19 & & & 19 & 15 & & & 16 & 18 & & & 16 & 8 & \\
\hline \multicolumn{17}{|l|}{ smoking } \\
\hline Yes & 383 & 191 & 192 & 0.004 & 0.519 & 65 & 177 & 9.265 & 0.002 & 54 & 189 & 0.308 & 0.329 & 48 & 193 & ( \\
\hline No & 103 & 51 & 52 & & & 38 & 206 & & & 49 & 194 & & & 55 & 190 & \\
\hline \multicolumn{17}{|l|}{$\mathrm{T}$ stage } \\
\hline 1 & 189 & 98 & 91 & 0.493 & 0.921 & 106 & 83 & 5.764 & 0.124 & 99 & 90 & 2.994 & 0.393 & 98 & 91 & \\
\hline 2 & 315 & 156 & 159 & & & 148 & 167 & & & 150 & 165 & & & 159 & 156 & \\
\hline 3 & 49 & 25 & 24 & & & 25 & 24 & & & 29 & 20 & & & 23 & 26 & \\
\hline 4 & 20 & 9 & 11 & & & 7 & 13 & & & 9 & 11 & & & 6 & 14 & \\
\hline \multicolumn{17}{|l|}{$\mathrm{N}$ stage } \\
\hline 1 & 364 & 177 & 187 & 5.822 & 0.121 & 179 & 185 & 2.004 & 0.572 & 180 & 184 & 1.057 & 0.787 & 192 & 172 & \\
\hline 2 & 108 & 63 & 45 & & & 53 & 55 & & & 59 & 49 & & & 43 & 65 & \\
\hline 3 & 87 & 40 & 47 & & & 44 & 43 & & & 42 & 45 & & & 42 & 43 & \\
\hline 4 & 2 & 2 & 0 & & & 0 & 2 & & & 1 & 1 & & & 0 & 2 & \\
\hline \multicolumn{17}{|l|}{ M stage } \\
\hline 0 & 387 & 195 & 192 & 1.501 & 0.155 & 187 & 10 & 0.401 & 0.337 & 193 & 12 & 0.000 & 0,578 & 195 & 9 & \\
\hline 1 & 24 & 9 & 15 & & & 200 & 14 & & & 194 & 12 & & & 192 & 15 & \\
\hline \multicolumn{17}{|l|}{ Disease stage } \\
\hline I & 308 & 151 & 157 & 2.956 & 0.398 & 156 & 152 & 0.843 & 0.839 & 152 & 156 & 1.176 & 0.759 & 164 & 144 & 4 \\
\hline II & 134 & 74 & 60 & & & 67 & 67 & & & 71 & 63 & & & 61 & 73 & \\
\hline III & 97 & 47 & 50 & & & 44 & 53 & & & 45 & 52 & & & 48 & 49 & \\
\hline IV & 28 & 11 & 17 & & & 14 & 14 & & & 15 & 13 & & & 10 & 18 & \\
\hline \multicolumn{17}{|l|}{ Overall } \\
\hline \multicolumn{17}{|l|}{ Survival Status } \\
\hline 0 & 355 & 180 & 175 & 0.430 & 0.258 & 188 & 95 & 2.962 & 0.050 & 182 & 99 & 0.800 & 0.210 & 188 & 93 & $\vdots$ \\
\hline 1 & 209 & 100 & 109 & & & 167 & 114 & & & 173 & 110 & & & 167 & 116 & \\
\hline Recurrence & & & & & & & & & & & & & & & & \\
\hline Free Survival & & & & & & & & & & & & & & & & \\
\hline Status & & & & & & & & & & & & & & & & \\
\hline 0 & 312 & 156 & 75 & 1.669 & 0.116 & 169 & 75 & 4.694 & 0.019 & 164 & 82 & 0.940 & 0.191 & 156 & 90 & ( \\
\hline 1 & 171 & 156 & 96 & & & 143 & 96 & & & 148 & 89 & & & 156 & 81 & \\
\hline
\end{tabular}

Table 2: The expression difference of IREB2, CHRNA5, CHRNA3, CHRNB4, HYKK, and PSMA4 genes between normal and lung adenocarcinoma tissues based on the TCGA database.

\begin{tabular}{|c|c|c|c|c|c|c|c|c|c|c|c|c|c|}
\hline & & \multicolumn{2}{|l|}{ IREB2 } & \multicolumn{2}{|c|}{ CHRNA5 } & \multicolumn{2}{|l|}{ CHRNA3 } & \multicolumn{2}{|c|}{ CHRNB4 } & \multicolumn{2}{|l|}{ HYKK } & \multicolumn{2}{|l|}{ PSMA4 } \\
\hline & & Normal & LAD & Normal & $\mathrm{LAD}$ & Normal & $\mathrm{LAD}$ & Normal & $\mathrm{LAD}$ & Normal & LAD & Normal & LAD \\
\hline Mean value & & 10.211 & 10.055 & 3.653 & 6.383 & 3.315 & 2.989 & 0.699 & 2.427 & 10.789 & 11.132 & 4.716 & 5.120 \\
\hline \multirow[t]{2}{*}{$95 \%$ confidence interval of the difference } & Lower & 0.087 & & -3.029 & & 0.051 & & -1.942 & & -0.451 & & -0.540 & \\
\hline & Upper & 0.225 & & -2.431 & & 0.600 & & -1.514 & & -0.235 & & -0.268 & \\
\hline T value & & 4.446 & & -18.106 & & 2.359 & & -15.968 & & -6.312 & & -5.871 & \\
\hline $\mathrm{P}$ value & & 0.000 & & 0.000 & & 0.021 & & 0.000 & & 0.000 & & 0.000 & \\
\hline
\end{tabular}


Table 3: The association between the expression of CHRNA5 and PSMA4 and clinical pathological features.

\begin{tabular}{|c|c|c|c|c|c|c|c|c|c|}
\hline \multirow[t]{2}{*}{ Characteristics } & \multirow[t]{2}{*}{$\mathrm{n}$} & \multicolumn{2}{|l|}{ CHRNA5 } & \multirow[t]{2}{*}{$\mathrm{X}^{2}$} & \multirow[t]{2}{*}{$P$} & \multicolumn{2}{|l|}{ PSMA4 } & \multirow[t]{2}{*}{$\mathrm{X}^{2}$} & \multirow[t]{2}{*}{$\mathrm{P}$} \\
\hline & & Low $\square 53 \square$ & High $\square 69]$ & & & Low $\square 50 \square$ & High $\square 72 \square$ & & \\
\hline \multicolumn{10}{|l|}{ Gender } \\
\hline Female & 65 & 32 & 33 & 1.897 & 0.116 & 27 & 38 & 0.018 & 0.521 \\
\hline Male & 57 & 21 & 36 & & & 23 & 34 & & \\
\hline \multicolumn{10}{|l|}{ Age } \\
\hline$<60$ & 44 & 21 & 23 & 0.589 & 0.745 & 18 & 26 & 0.848 & 0.654 \\
\hline $60-80$ & 75 & 31 & 44 & & & 30 & 45 & & \\
\hline$>80$ & 3 & 1 & 2 & & & 2 & 1 & & \\
\hline \multicolumn{10}{|l|}{ smoking } \\
\hline Yes & 40 & 18 & 22 & 0.030 & 0.510 & 15 & 25 & 0.346 & 0.351 \\
\hline No & 67 & 29 & 38 & & & 29 & 38 & & \\
\hline \multicolumn{10}{|l|}{ T stage } \\
\hline 1 & 62 & 31 & 31 & 9.293 & 0.026 & 26 & 36 & 0.923 & 0.820 \\
\hline 2 & 40 & 10 & 30 & & & 16 & 24 & & \\
\hline 3 & 11 & 4 & 7 & & & 3 & 8 & & \\
\hline 4 & 2 & 2 & 0 & & & 1 & 1 & & \\
\hline \multicolumn{10}{|l|}{$\mathrm{N}$ stage } \\
\hline 1 & 81 & 35 & 46 & 2.282 & 0.320 & 32 & 49 & 0.671 & 0.715 \\
\hline 2 & 22 & 7 & 15 & & & 9 & 13 & & \\
\hline 3 & 18 & 10 & 8 & & & 9 & 9 & & \\
\hline 4 & 0 & 0 & 0 & & & 0 & 0 & & \\
\hline \multicolumn{10}{|l|}{ M stage } \\
\hline 0 & 118 & 52 & 66 & 2.318 & 0.182 & 50 & 68 & 2.166 & 0.198 \\
\hline 1 & 3 & 0 & 3 & & & 0 & 3 & & \\
\hline \multicolumn{10}{|l|}{ Disease stage } \\
\hline I & 1 & 1 & 0 & 3.817 & 0.421 & 0 & 1 & 1.360 & 0.715 \\
\hline II & 71 & 28 & 43 & & & 27 & 44 & & \\
\hline III & 25 & 9 & 16 & & & 11 & 14 & & \\
\hline IV & 19 & 10 & 9 & & & 9 & 10 & & \\
\hline \multicolumn{10}{|c|}{ Overall Survival Status } \\
\hline 0 & 90 & 44 & 46 & 6.807 & 0.007 & 40 & 50 & 7.094 & 0.006 \\
\hline 1 & 22 & 4 & 18 & & & 3 & 19 & & \\
\hline \multicolumn{10}{|c|}{ Progress Free Survival Status } \\
\hline 0 & 78 & 38 & 40 & 5.744 & 0.013 & 34 & 44 & 6.807 & 0.007 \\
\hline 1 & 30 & 7 & 23 & & & 5 & 25 & & \\
\hline
\end{tabular}

\section{Figures}


IREB2

7\% II: -

CHRNA5

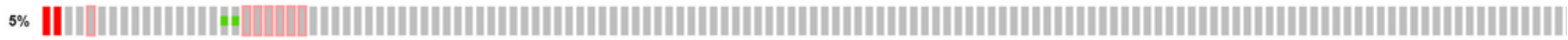

CHRNA3

5\%

CHRNB4

7\%

HYKK

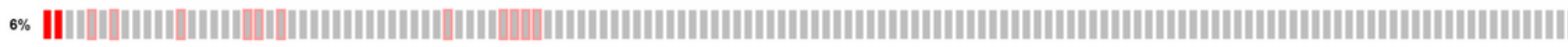

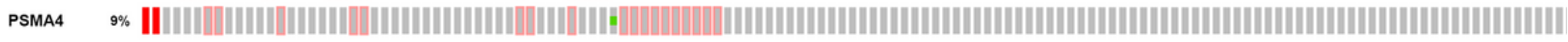

IREB2

CHRNA5

CHRNA3

CHRNB4

HYKK

PSMA4

Genetic Alteration

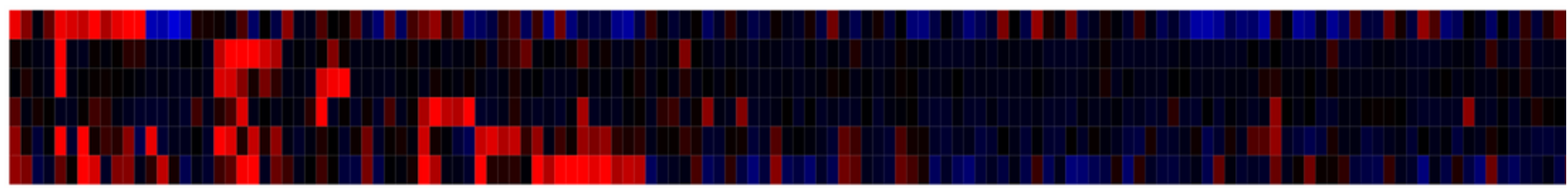

|| Inframe Mutation (unknown significance) || Missense Mutation (unknown significance) || Truncating Mutation (unknown significance) | Amplification

II mRNA Upregulation || mRNA Downregulation || No alterations

Expression Heatmap

3

Figure 1

The mutation of IREB2, CHRNA5, CHRNA3, CHRNB4, HYKK,and PSMA4 genes in lung adenocarcinoma based on the TCGA database.

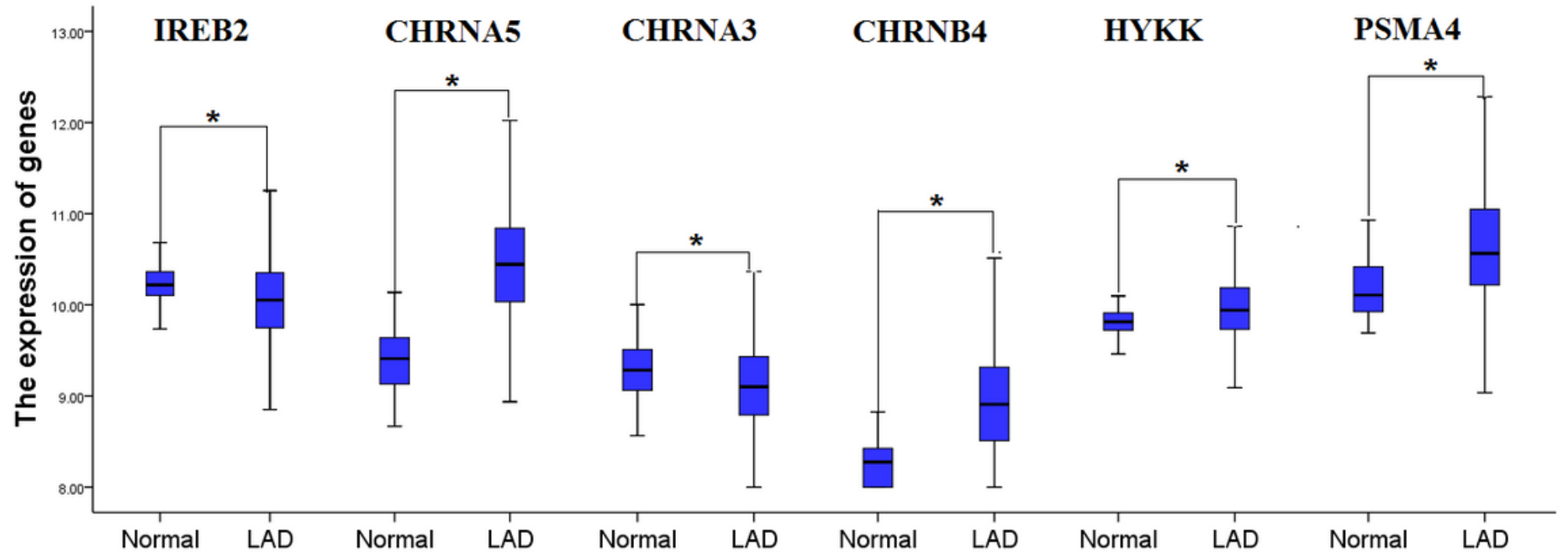

Figure 2

The expression of IREB2, CHRNA5, CHRNA3, CHRNB4, HYKK,and PSMA4 genes between normal and lung adenocarcinoma tissues based on the TCGA database. 

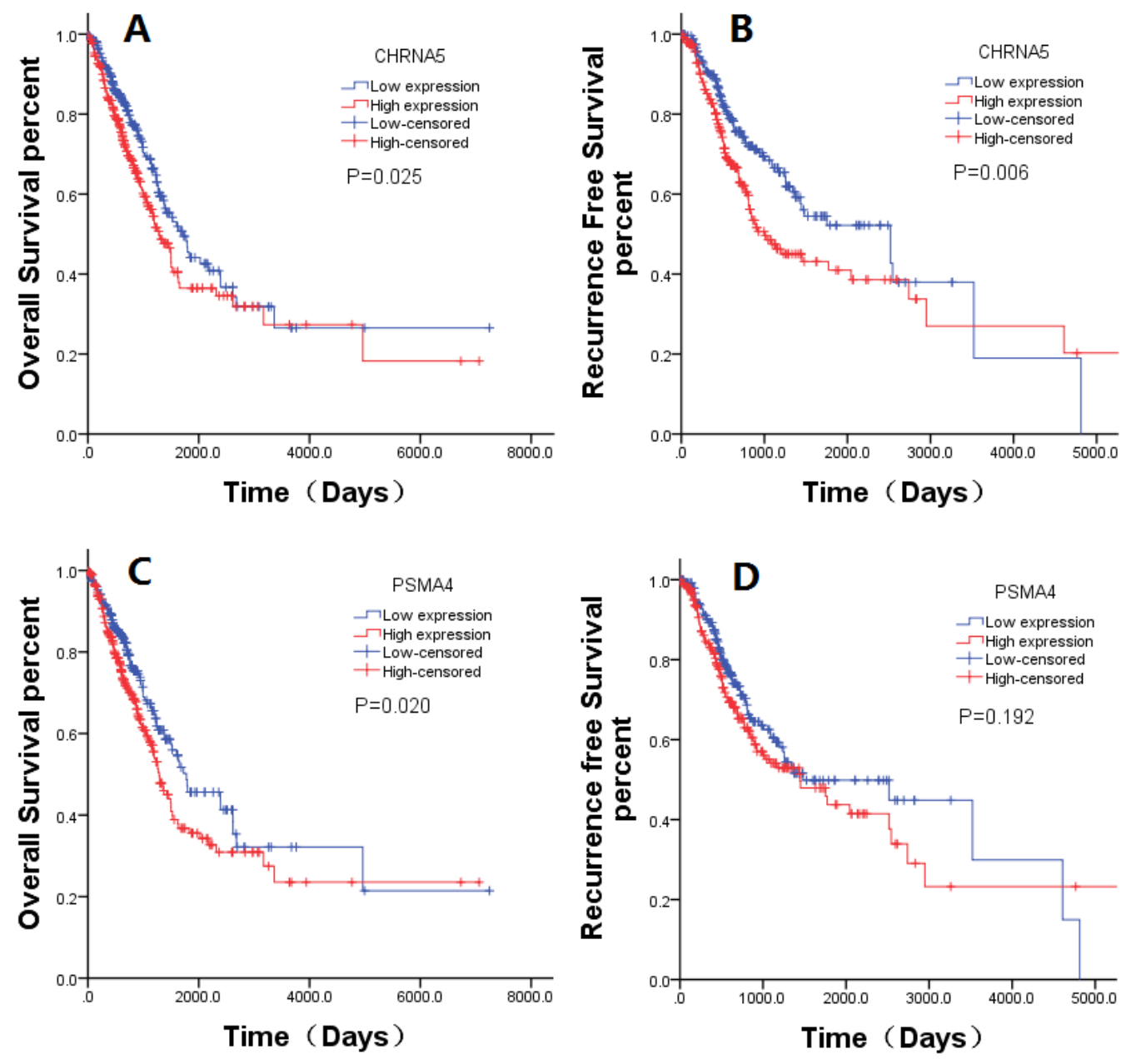

Figure 3

Kaplan-Meier analysis of the correlation between CHRNA5, PSMA4 expression level and overall survival (OS) / recurrence free survival (RFS) based on the TCGA database. 
A
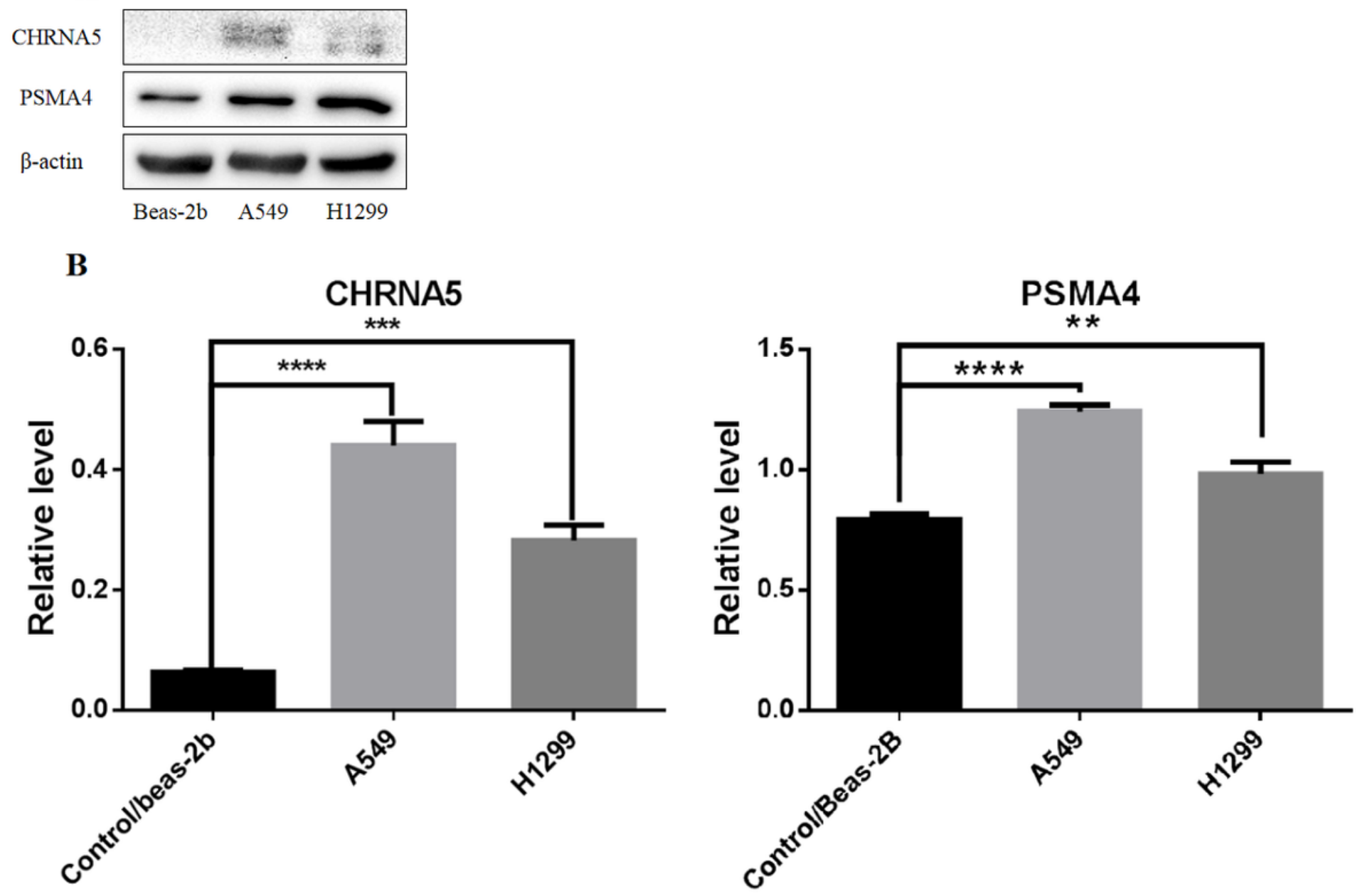

Figure 4

The expression of CHRNA5 and PSMA4 in Beas-2b, A549 and H1299 cells. The expression of CHRNA5 and PSMA4 in A549 and H1299 cells were obvious higher than Beas-2b cell.A. The Western blotting result of the expression of CHRNA5 and PSMA4; $B$. The relative levels of the expression of CHRNA5 and PSMA4 based on The Western blotting.*** means that the $\mathrm{P}<0.001$; ${ }^{\star \star \star *}$ means that the $\mathrm{P}<0.0001$.

Normal

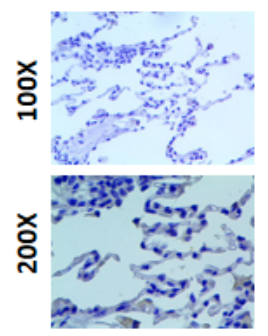

CHRNA5

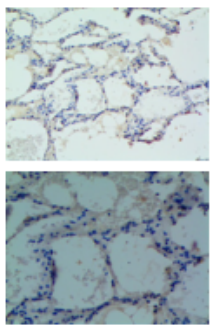

PSMA4

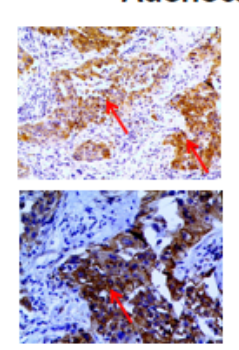

CHRNA5

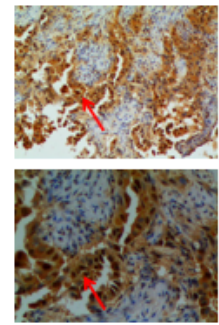

PSMA4

Figure 5

Expression of CHRNA5 and PSMA4 in lung adenocarcinoma. The expression of CHRNA5 and PSMA4 in the lung tissue were located in the cytoplasm, and the expression of CHRNA5 and PSMA4 were higher in lung adenocarcinoma tissues, and lower in normal tissues. The results showed that the figure was upper 100 * and below 200 *. 

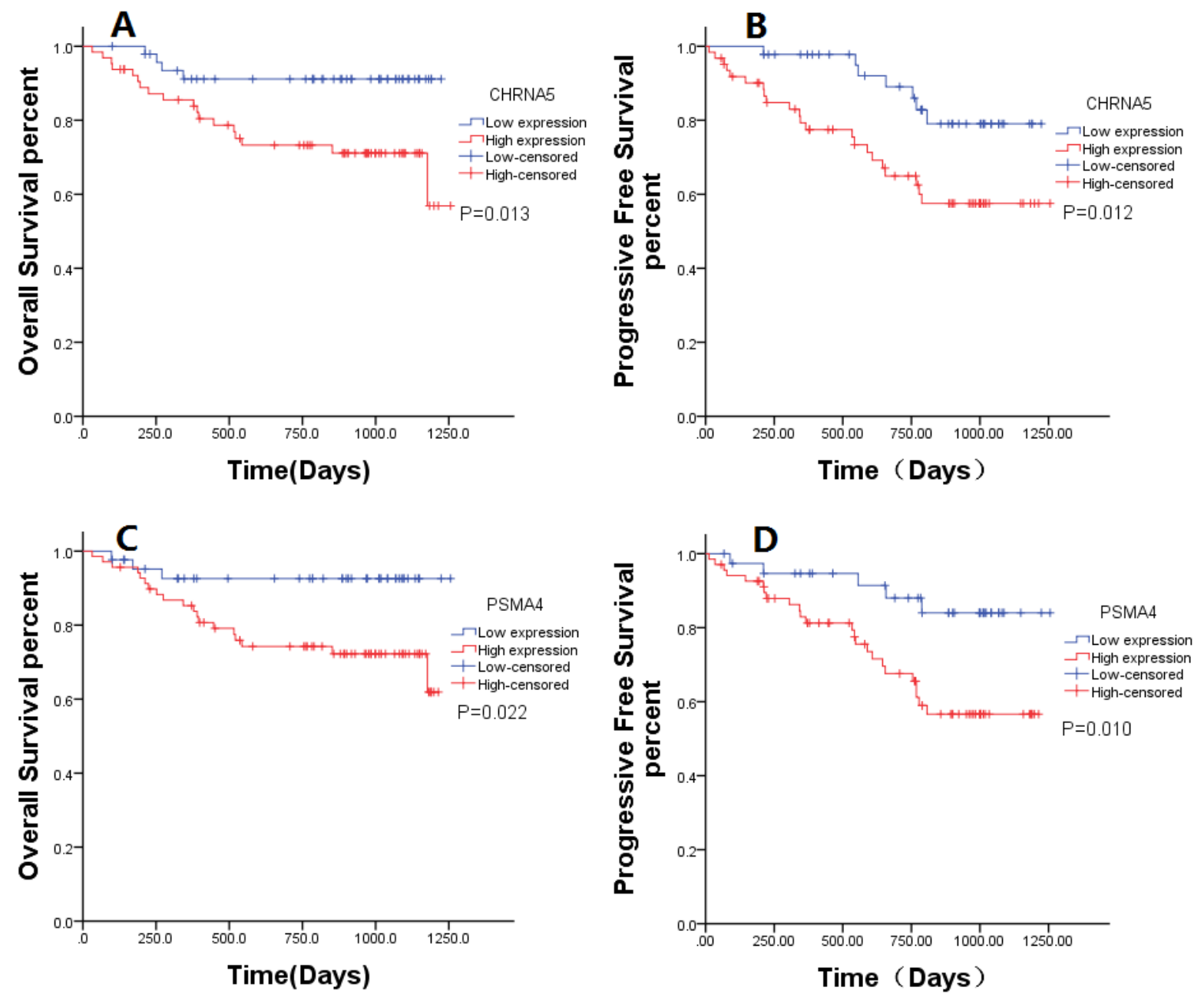

Figure 6

Kaplan-Meier analysis of the correlation between CHRNA5, PSMA4 expression level and overall survival (OS) / recurrence free survival (RFS) based on the clinical data. 

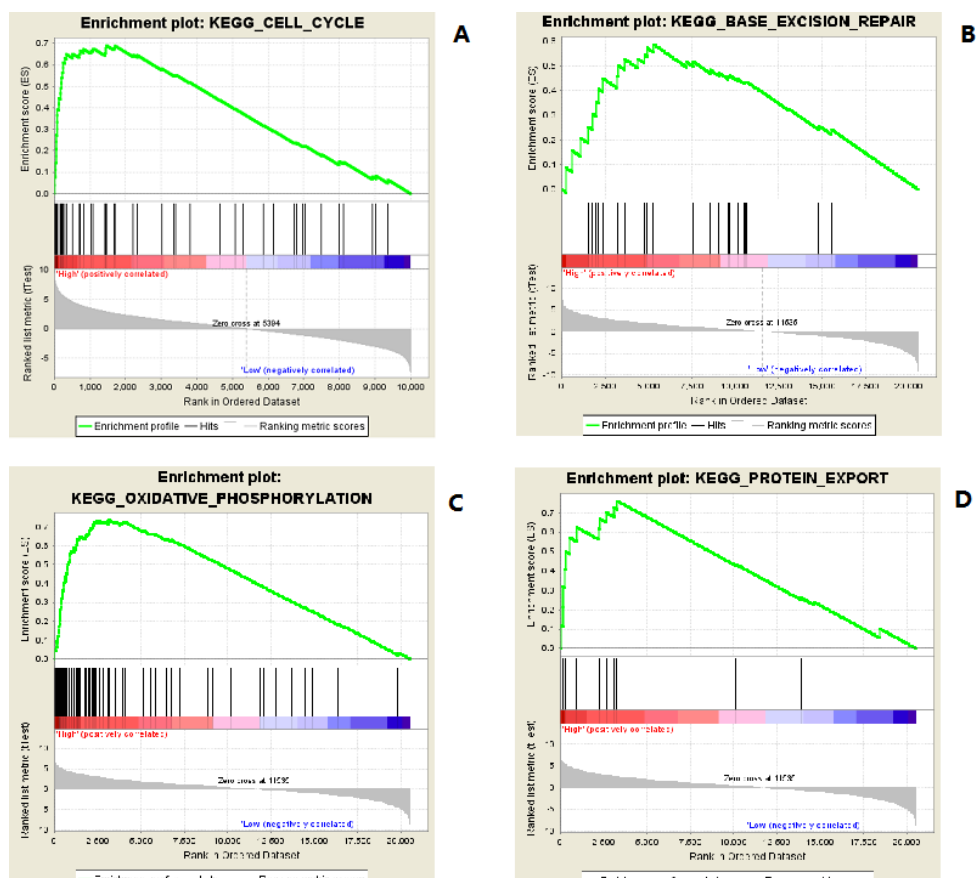

C
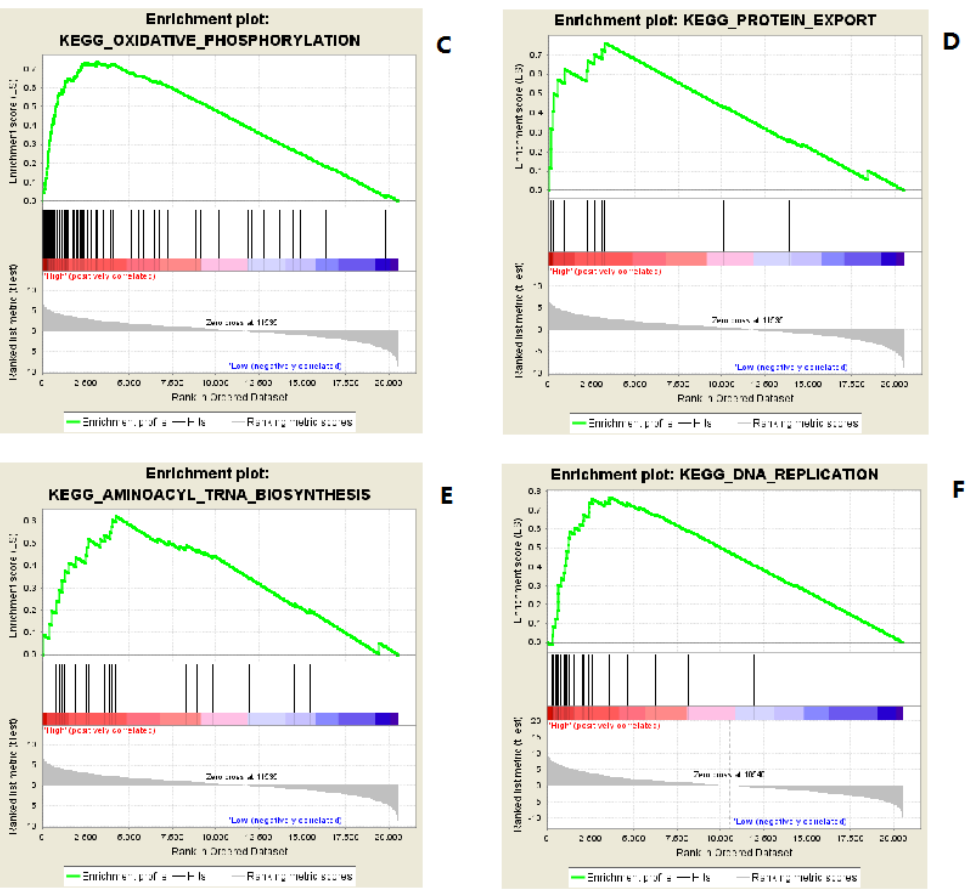

\section{Figure 7}

GSEA with high expression of CHRNA5, HYKK and PSMA4. The results of GSEA method suggested that CHRNA5 high expression samples were enriched to cell cycle, base excision repair, oxidative phosphorylation, protein export, aminoacyl tRNA biosynthesis.

\section{Supplementary Files}

This is a list of supplementary files associated with this preprint. Click to download.

- Supplement.docx 